\title{
Simulation of PWM DC-DC converters using eigenvalues and eigenvectors
}

\begin{abstract}
Aleksandra Lekić
In this paper, a time-domain simulation of the DC-DC converters' dynamic behavior is described. Corresponding to the state-space model for each possible operation mode of the circuit, an analytic solution is provided. Converter's regulator is discretized using the pole-zero placement technique based on its Laplace transfer function. An algorithm results in a very accurate simulation due to the exact solution of the circuit's equations.
\end{abstract}

K e y w or d s: simulation, DC-DC converters, PWM, eigenvalues

\section{Introduction}

Simulation of power electronics' circuits has been a research topic over the years [1]. Power electronics' circuits are built as switching circuits which makes their simulation very challenging [2]. Furthermore, the switching power converters' simulators can be classified into general purpose, piecewise-linear and averaged simulators.

General purpose simulators are developed for simulation of the wide range of electronic circuits [3]. From power electronics' point of view, due to the existence of switches, general purpose simulator Spice [3] and its derivations being LTspice, HSPICE, etc. are shown to have convergence issues. These problems are caused by very complex models of switches, realized as transistors and diodes, and from their very fast switching being much faster than the line voltage frequency $f_{S} \gg f_{0}$. Sometimes the convergence problems can be bypassed by decreasing the simulation time step. However, there are cases when a simulation cannot be performed although the simulation step is very small (for example, PFC converters).

Convergence problems in general purpose simulators motivated the development of other simulators especially made for simulating switching converter's power stages. Piecewise-linear simulators [4] assume a switching converter with its linearized model for each state of the switch, being on or off. However, not every type of switching is possible in circuits and the operation state (mode) of the converter cannot be easily checked. Operation mode determination causes in piecewise-linear simulators problems similar to the previously mentioned convergence problems.

Development of the averaged simulators, which are developed for power converter's circuits with averaged voltages and currents in the circuit, is based on slow variations of the circuit's input voltage or current comparing to the switching frequency. Averaging of the voltages and currents in the circuit is often done on the level of a switching cell and as the state-space averaging. Switching cell averaging is made by representing a converter as a two port network consisting of inductors, capacitors and switches [5]. A lot of the research effort has been done in order to determine and classify switching cells; see [57] for more details. However, averaging on the level of a switching cell has to be carefully done in all of the operating modes of the converter being continuous and discontinuous ones. Another type of averaging simulators is based on the state-space averaging. State-space averaging is based on developing state-space system models and then averaging them as described in [8-10].

In this paper an algorithm for the transient simulation of the pulse width modulated (PWM) DC-DC converters, is presented. This algorithm is based on the piecewise simulation of the exact converter model described with ordinary differential equations for the state variables. Ordinary differential equations (ODEs) are solved using eigenvectors and eigenvalues of the system matrix [11] and then the homogeneous and particular solutions of the system are found. The outcome solution of the ODEs is exact without any approximations, thus resulting in this algorithm being very accurate and providing an option for increasing the simulation's time step. However, there is still a problem for determination operating modes which is solved using the predefined switching mode as will be described in Section 2. Due to development of microcontrollers and digital control [12], the regulation of the converter is shown to be suitable for digital realization. In that manner, the converter regulation is realized in discrete-time.

The paper is organized as follows. Section 2 provides the development of the circuit state-space models and the solutions of ODEs, the regulation design and the determination of the circuit's operation modes. In Section 3, the program implementation is described. Simulation examples of practical importance are provided in Section 4. Finally, some concluding remarks are given in Section 5.

\footnotetext{
* School of Electrical Engineering, University of Belgrade, Belgrade, Serbia, lekic.aleksandra@etf.rs
} 


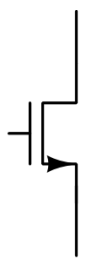

(a)

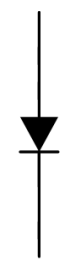

(b)

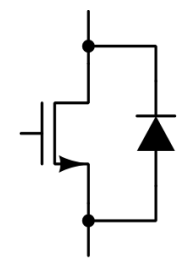

(c)

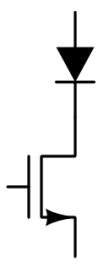

(d)
Fig. 1. Switches realization: (a) - transistor, (b) - a diode, (c) current bidirectional and (d) — voltage bidirectional

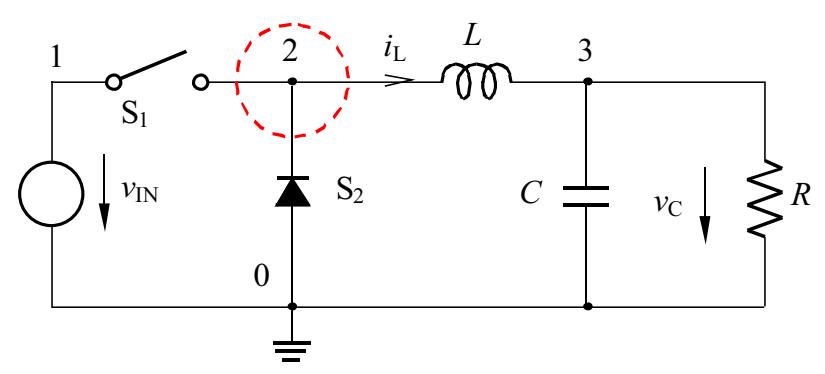

Fig. 2. Buck converter

\section{Transient Simulation - Constraints}

In this section, the simulation algorithm and its implementation are explained in detail.

\subsection{Formulation of circuit equations}

DC-DC converters are the switching circuits consisting of two or more switches. Most of the families of DCDC converters have even number of switches [13]. Each switch has two operation modes: on and off. Switches are classified according to the polarity of the current which flows through the switch when it is on and the polarity of voltage in the off state of the switch. The possible realizations are one-quadrant, two-quadrant and fourquadrant switches [2]. As can be seen in Figs. 1a and $1 \mathrm{~b}$ possible ways for realization of a one-quadrant switch is to use a transistor or a diode, two-quadrant switches are current (Fig. 1c) and voltage bidirectional (Fig. 1d) and four-quadrant is a combination of the previous two two-quadrant switches (current and voltage bidirectional switch). Also, switches can be controllable or not. Controllable switches are the ones which have a transistor. In Fig. 1 controllable switches are transistor, current and voltage bidirectional.

In Fig. 2, the Buck converter is depicted, which represents one of the common DC-DC converters besides Boost and Buck-Boost converter. As can be seen from Fig. 2, Buck converter has two switches, which provides four operation states of the circuit. However, not all of the states are possible. For example state when both switches are on makes the independent voltage source $v_{I N}$ shortconnected which is not valid and makes this state impossible.
Determination of the valid converter's operation modes can be done in a few steps. After defining all switches' states, first the states that are not possible when the desired control is applied, are eliminated. In the second step, the loops consisting only of switches and voltage sources, and cutsets of switches and current sources, are determined. The loops consisting only of switches and voltage sources result in a short-connected independent voltage source when all switches in the loop are on. In the cutsets consisting of switches and current sources, the current sources would be disconnected if all the switches in the cutset are off. These states are clearly not valid and thus eliminated.

The third step is to find the states which can produce one of the discontinuous conduction modes: discontinuous inductor current mode (DICM) or discontinuous capacitor voltage mode (DCVM). This is necessary for the circuit equations' formulation. According to [14] there can only be two continuous states and some number of discontinuous states which is not bigger than the number of state variables ( $i e$ inductors and capacitors). In [15] it is described that DICM can occur if in the circuit exists a cutset consisting only of current sources, inductors and switches, denoted as DICM cutsets. The current of this DICM cutset is given as

$$
\sum_{i} \pm i_{L i}+\sum_{j} \pm i_{S j}+\sum_{k} \pm i_{G k}=0
$$

where $i_{L i}$ are inductors' currents, $i_{S j}$ are switches' currents and $i_{G k}$ are independent current sources' currents. Converter enters DICM when the sum of the switches' currents reaches zero which forces the switches to turn off. In general case, as shown in [16], during the DICM, the sum of inductors' voltages is equal zero, but their current has some nonzero value which is nearly constant during the whole DICM interval.

A similar scenario is with DCVM which occurs when in the converter exists a loop with switches, capacitors and independent voltage sources when all switches in DCVM loop are closed (in the remaining text denoted as DCVM loops). The sum of the voltages in the DCVM loop is

$$
\sum_{i} \pm v_{C i}+\sum_{j} \pm v_{S j}+\sum_{k} \pm v_{G k}=0,
$$

where $v_{C i}$ are capacitors' voltages, $v_{S j}$ are voltages across switches and $v_{G k}$ are voltages of independent voltage sources. Equality of the sum of capacitor voltages to the sum of independent voltage sources' voltages, since $\sum_{j} \pm v_{S j}=0$ for all switches closed, is the condition for entering DCVM.

Circuit equations are formed by the modified nodal analysis [17] which is defined as

$$
H_{x}=\boldsymbol{z},
$$

where $\mathbf{x}$ presents a vector consisting of node voltages, currents through independent voltage sources and currents through switches, as well as derivatives of inductor 


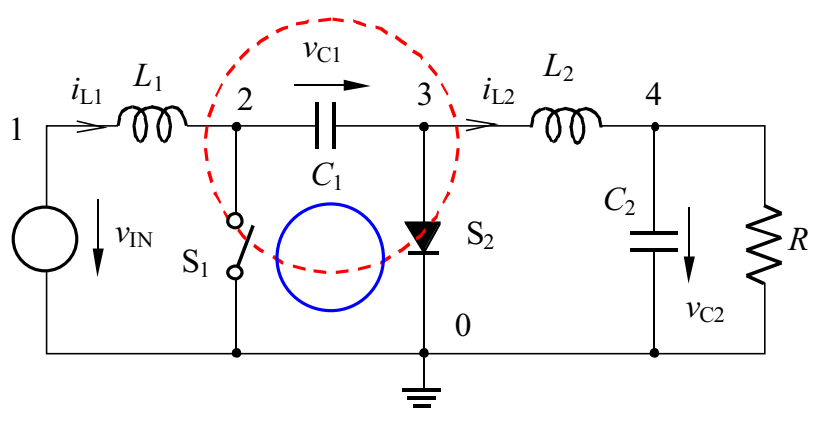

Fig. 3. Ćuk converter

currents and derivatives of capacitor voltages. Vector $\boldsymbol{z}$ consists of independent sources' values, inductor currents and capacitor voltages, while matrix $\mathbf{H}$ contains quantities. In order to solve the converter's circuit containing switches, an extra equation for each state of the switch, on and off state, must be added as

$$
\begin{array}{ll}
i_{S}=0 & \text { for S off, } \\
v_{S}=0 & \text { for S on. }
\end{array}
$$

The equations for reactive elements in the circuit, inductor and capacitor, are similar to the equations for independent current and voltage source, respectively. Therefore, an extra equation for the inductor in the example of Buck converter shown in Fig. 2, is given as

$$
\frac{\mathrm{d} i_{L}}{\mathrm{~d} t}=\frac{v_{2}}{L}-\frac{v_{3}}{L}
$$

and for capacitor

$$
v_{3}=v_{C}
$$

For the example including the Buck converter in the state when switch S1 is on and S2 (switch S2 represents a diode which is a non-controllable switch) is off, the system of equations (3) is as follows:

$$
\left[\begin{array}{rrrrrrrr}
0 & 0 & 0 & 1 & 1 & 0 & 0 & 0 \\
0 & 0 & 0 & 0 & -1 & 0 & 0 & 0 \\
0 & 0 & \frac{1}{R} & 0 & 0 & 0 & 0 & C \\
1 & 0 & 0 & 0 & 0 & 0 & 0 & 0 \\
1 & -1 & 0 & 0 & 0 & 0 & 0 & 0 \\
0 & 0 & 0 & 0 & 0 & 1 & 0 & 0 \\
0 & -\frac{1}{L} & \frac{1}{L} & 0 & 0 & 0 & 1 & 0 \\
0 & 0 & 1 & 0 & 0 & 0 & 0 & 0
\end{array}\right]\left[\begin{array}{c}
v_{1} \\
v_{2} \\
v_{3} \\
i_{I N} \\
i_{S 1} \\
i_{S 2} \\
\frac{\mathrm{d} i_{L}}{\mathrm{~d} t} \\
\frac{\mathrm{d} v_{C}}{\mathrm{~d} t}
\end{array}\right]=\left[\begin{array}{c}
0 \\
-i_{L} \\
i_{L} \\
v_{I N} \\
0 \\
0 \\
0 \\
v_{C}
\end{array}\right]
$$

When the converter operates in a discontinuous mode, DICM or DCVM, some modifications in the equations are made. In general, sum of inductor currents or capacitor voltages is constant. The discontinuous mode enables the system reduction by one equation in the form of cutset/loop equations. The equation for DICM cutset (1) is reduced to

$$
\sum_{i} \pm i_{L i}+\sum_{k} \pm i_{G k}=0
$$

and for DCVM loop (2)

$$
\sum_{i} \pm v_{C i}+\sum_{j} \pm v_{G j}=0 .
$$

Thus, DCVM and DICM provide one extra equation by including dependence of capacitor voltages or inductor currents. On the other hand one of the unknown state variables is symbolically changed to the combination of sums of inductor currents and current sources currents/capacitor voltages and voltage sources voltages.

A common case for DICM is when inductor current becomes zero which means that DICM cutset contains only one inductor and switches and possibly current sources. Then the equation is modified by setting inductor voltage to zero. In Fig. 2, DICM cutset is depicted as red dashed circle and as it can be seen this cutset contains one inductor and two switches. Other, more complicate case, is when the number of inductors in the DICM cutset is greater than one. From Fig. 3 it can be seen that this kind of DICM occurs in the Cuk converter where DICM cutset is depicted as red dashed circle and crosses two inductors and two switches. The equation for DICM cutset for the Ćuk converter in DICM is given by

$$
i_{L 1}-i_{L 2}=0
$$

and therefore current $i_{L 1}$ is replaced by $-i_{L 2}$ inside vector $\boldsymbol{z}$. An extra equation which has to be added is then

$$
\frac{\mathrm{d} i_{L 1}}{\mathrm{~d} t}-\frac{\mathrm{d} i_{L 2}}{\mathrm{~d} t}=0
$$

When all possible states in which DC-DC converter can operate are formulated, it is important to determine the switching between the states. According to [13] PWM converter can have two continuous conduction modes and a number of discontinuous conduction modes. They can operate in discontinuous quasi resonant mode (DQRM) when both discontinuous modes, DICM and DCVM, occur during one switching interval.

For PWM converters possible transitions are as follows:

- $01 \rightarrow 10$,

- $01 \rightarrow 10 \rightarrow 00$

- $01 \rightarrow 11 \rightarrow 10$.

The states are represented as binary variables where $2^{0}$ bit represents controllable switch $\mathrm{S} 1$ equal to 1 during interval $n T_{S} \leq t<(n+d) T_{S}$ and $2^{1}$ bit is $\overline{\mathrm{S} 2}$ with possible realization as a diode. In the case when DQRM can occur, which means that in the circuit exist both a DICM cutset and a DCVM loop, in addition it is possible to have transition $01 \rightarrow 11 \rightarrow 10 \rightarrow 00$. 


\subsection{Solving State-Space Equations Using Eigenvalues}

A system consisting of ordinary differential equations can be presented in the standard state-space form as follows:

$$
\begin{aligned}
& \dot{\mathbf{x}}=\mathbf{A} \mathbf{x}+\mathbf{B} \boldsymbol{u}, \\
& \boldsymbol{y}=\mathbf{C}_{\boldsymbol{x}}+\mathbf{D} \boldsymbol{u}
\end{aligned}
$$

where $\mathbf{x}$ is the vector which consists of state variables, i.e., inductor currents and capacitor voltages, $\boldsymbol{u}$ is the vector of independent sources and $\boldsymbol{y}$ is the vector of output variables [18]. In this work only DC-DC PWM converters with one input and one output, as described in $[6,5]$, will be considered. Let us denote the number of inductors in the converter as $m$ and the number of capacitors as $n$. Generally, the number of inductors and the number of capacitors doesn't have to be equal. However, in order to obtain independent output voltage of the load resistance, it is shown that the total number of inductors should be equal to the total number of capacitors, $m=n$ [19]. The switching cell is considered as a three-port element between input and output of the converter and it is made of switches, inductors and capacitors.

Depending on the switch realization and the switching cell network during one switching interval, a number of subintervals may occur. However, that number is equal to $2+k$, where 2 subintervals are usual CCM intervals and $k$ represents different number of discontinuous modes, as in [14]. During one switching period consisting of $2+k$ subintervals, the state-space models are

$$
\dot{\mathbf{x}}(t)=\left\{\begin{array}{c}
\mathbf{A}_{1} \mathbf{x}(t)+\mathbf{B}_{1} \mathbf{u}(t), \quad n T_{S} \leq t<\left(n+d_{1}\right) T_{S} \\
\mathbf{A}_{2} \mathbf{x}(t)+\mathbf{B}_{2} \mathbf{u}(t), \\
\left(n+d_{1}\right) T_{S} \leq t<\left(n+d_{1}+d_{2}\right) T_{S} \\
\ldots \\
\mathbf{A}_{k+2} \mathbf{x}(t)+\mathbf{B}_{k+2} \mathbf{u}(t), \\
\quad\left(n+\sum_{j=1}^{k+1} d_{j}\right) T_{S} \leq t<(n+1) T_{S}
\end{array}\right.
$$

and

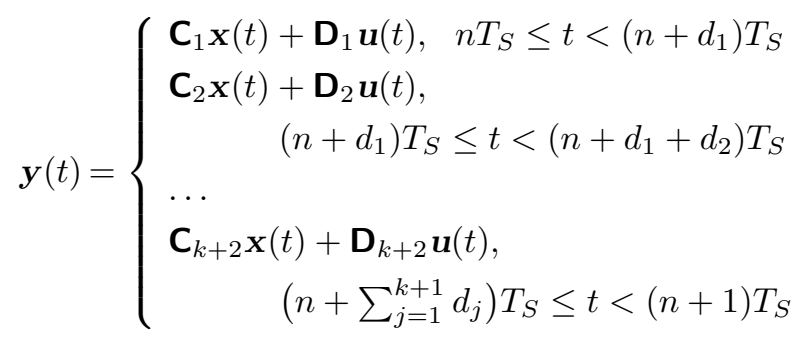

In this work all of the discontinuous modes are considered [15]. Again, $m$ is the number of inductors in the circuit, $n$ is the number of capacitors, $p$ is the number of independent sources and $q$ is the number of output variables. Earlier defined vectors and matrices are of dimensions $\mathbf{A}_{(m+n) \times(m+n)}, \mathbf{B}_{(m+n) \times p}, \mathbf{C}_{q \times(m+n)}$ and $\mathbf{D}_{q \times p}$.

State-space differential equations have the solution of the form

$$
\mathbf{x}(t)=\mathbf{x}_{p}(t)+\mathbf{x}_{h}(t)
$$

where $\boldsymbol{x}_{p}$ is the particular and $\boldsymbol{x}_{h}$ is the homogeneous solution. Homogeneous solution is found by calculating eigenvalues of the system matrix A from equation (10). Matrix $\mathbf{A}$ has $(m+n)$ rows and columns, so there are $m+n$ eigenvalues, $\underline{\lambda}_{j} \in \mathbb{C}$ where $j \in\{1,2, \ldots, m+n\}$. In the case of DC-DC converters, eigenvalues are either zero or distinct real or complex numbers. In the case when there are complex eigenvalues, they are in conjugatecomplex pairs because their characteristic polynomial has real coefficients. The homogeneous solution in the case of distinct eigenvalues is given by

$$
\mathbf{x}_{h}(t)=\sum_{i=1}^{m+n} \underline{\mathbf{x}}_{i} \mathrm{e}^{\underline{\lambda}_{i}\left(t-t_{0}\right)}=\sum_{i=1}^{m+n} \underline{\mathbf{X}}_{i} \mathrm{e}^{\underline{\lambda}_{i} t},
$$

where $t=t_{0}$ denotes the beginning of the interval. Thus, for each interval during one switching period, the homogeneous solution can be analytically solved by calculating system eigenvalues.

The particular solution is obtained by solving the following equation

$$
\mathbf{A} \mathbf{x}_{p}(t)+\mathbf{B} \mathbf{u}(t)=0 \text {. }
$$

If matrix $\mathbf{A}$ is invertible, particular solution is equal to

$$
\mathbf{x}_{p}(t)=-\mathbf{A}^{-1} \mathbf{B} \mathbf{u}(t) \text {. }
$$

However, the system matrix can have zero eigenvalue, meaning being singular. If matrix $\mathbf{A}$ has zero eigenvalues, then the particular solution can be determined as

$$
\left(\mathbf{P}^{-1} \mathbf{Q} \mathbf{u}(t)\right)^{\top}
$$

where matrix $\mathbf{P}$ is obtained from matrix $\mathbf{A}$ by the elimination of rows and columns which are containing only zeros or that are linear combination of other rows/columns. This occurs in the case of discontinuous modes and in some continuous conduction modes as well. Vector $\mathbf{Q}$ is obtained from vector $\mathbf{B}$ by elimination the same rows that are eliminated in matrix $\mathbf{A}$. The particular solution for the state variables whose equations are eliminated in equation (18) are their values from the end of the previous time interval.

Assuming that $t=t_{0}$ denotes the end of the previous and the beginning of the next time interval, the value of the state variables at the beginning of the interval is known and denoted as $\mathbf{x}\left(t_{0}\right)$. Using previously formulated equations (10), the system of $m+n$ equations can be written as

$$
\begin{gathered}
\mathbf{x}\left(t_{0}\right)=\mathbf{x}_{p}\left(t_{0}\right)+\sum_{i=1}^{m+n} \underline{\mathbf{x}}_{i} \mathrm{e}^{\underline{\lambda}_{i} t_{0}}, \\
\dot{\mathbf{x}}\left(t_{0}\right)=\dot{\mathbf{x}}_{p}\left(t_{0}\right)+\sum_{i=1}^{m+n} \underline{\lambda}_{i} \underline{\mathbf{x}}_{i} \mathrm{e}^{\underline{\lambda}_{i} t_{0}}=\mathbf{A} \mathbf{x}\left(t_{0}\right)+\mathbf{B} \mathbf{u}\left(t_{0}\right), \\
\vdots \\
\boldsymbol{x}^{(m+n-1)}\left(t_{0}\right)=\mathbf{x}_{p}^{(m+n-1)}\left(t_{0}\right)+\sum_{i=1}^{m+n} \underline{\lambda}_{i}^{m+n-1} \underline{\mathbf{x}}_{i} \mathrm{e}^{\underline{\lambda}_{i} t_{0}} \\
=\mathbf{A}^{m+n-1} \mathbf{x}\left(t_{0}\right)+\sum_{j=1}^{m+n-1} \mathbf{A}^{m+n-1-j} \mathbf{B} \mathbf{u}^{(j-1)}\left(t_{0}\right),
\end{gathered}
$$


where $\underline{\mathbf{X}}_{i}$ are spectral components of all state variables corresponding to eigenvalue $\underline{\lambda}_{i}$. In DC-DC converters, the vector of independent sources $\boldsymbol{u}$ is constant or slowly changing during one switching period. Thus, all derivatives $\mathbf{u}^{(j-1)}\left(t_{0}\right), j \geq 1$ will be considered as zero and omitted in what follows. Derivatives of the partial solution are zero, because they are linear combinations of independent sources values. The solution of equations (19) provides quantities for further calculation which can be found using the reduced row echelon form [11] of the system as

$$
\mathbf{M}\left[\begin{array}{c}
\underline{\mathbf{x}}_{1} \\
\underline{\mathbf{x}}_{2} \\
\vdots \\
\underline{\mathbf{x}}_{m+n}
\end{array}\right]=\left[\begin{array}{c}
\mathbf{x}\left(t_{0}\right)-\mathbf{x}_{p} \\
\mathbf{A} \mathbf{x}\left(t_{0}\right)+\mathbf{B} \mathbf{u} \\
\vdots \\
\mathbf{A}^{(m+n-1)} \mathbf{x}\left(t_{0}\right)+\mathbf{A}^{(m+n-2)} \mathbf{B u}
\end{array}\right]
$$

where matrix $\mathbf{M}_{(m+n)^{2} \times(m+n)^{2}}$ consists of $(m+n)^{2}$ matrices indexed as $M_{i, j}$ as follows:

$$
\mathbf{M}=\left[\begin{array}{cccc}
\mathbf{M}_{0,1} & \mathbf{M}_{0,2} & \ldots & \mathbf{M}_{0, m+n} \\
\mathbf{M}_{1,1} & \mathbf{M}_{1,2} & \ldots & \mathbf{M}_{1, m+n} \\
\vdots & \vdots & & \vdots \\
\mathbf{M}_{m+n-1,1} & \mathbf{M}_{m+n-1,2} & \ldots & \mathbf{M}_{m+n-1, m+n}
\end{array}\right]
$$

Matrices $\mathbf{M}_{i, j}$ have $(m+n)$ rows and columns and

$$
\mathbf{M}_{i, j}=\operatorname{diag}\left\{\underline{\lambda}_{j}^{i} \mathrm{e}^{\underline{\lambda}_{j} t_{0}}\right\}
$$

Equations (19) and (20) can be rewritten for the case of singular system matrix A. System matrix is singular in all discontinuous conduction modes and in some continuous conduction modes, eg, continuous conduction modes of the Ćuk converter (see Appendix 2). Let us assume that the number of zero eigenvalues is $r$. That means that the column connected matrices $\mathbf{A}$ and $\mathbf{B}$ have $r$ zero rows or rows that are some linear combination of other rows. Therefore, $r$ equations can be eliminated and degenerated system of equations is then of dimension $r(m+n)$.

The solution for the described problem is to eliminate eigenvalues equal to zero while calculating spectral components $\underline{\mathbf{X}}_{i}$ and to reduce the order of the system. In the case of $r$ zero eigenvalues, the number of nonzero eigenvalues is $m+n-r$, and the originally system (20) becomes

$$
\begin{aligned}
& \mathbf{M}\left[\begin{array}{c}
\underline{\mathbf{X}}_{1} \underline{\mathbf{X}}_{2} \\
\vdots \\
\underline{\mathbf{x}}_{m+n-r}
\end{array}\right]= \\
& {\left[\begin{array}{c}
\mathbf{x}\left(t_{0}\right)-\mathbf{x}_{p}\left(t_{0}\right) \\
\mathbf{A x}\left(t_{0}\right)+\mathbf{B u}\left(t_{0}\right) \\
\vdots \\
\mathbf{A}^{(m+n-r-1)} \mathbf{x}\left(t_{0}\right)+\mathbf{A}^{(m+n-r-2)} \mathbf{B u}\left(t_{0}\right)
\end{array}\right]}
\end{aligned}
$$

with matrix $\mathbf{M}_{(m+n)(m+n-r) \times(m+n)(m+n-r)}$. It should be mentioned that $r$ elements' values can be formulated as linear combinations of the remaining $m+n-r$ equations. Equation (21) shows that even for those elements $m+n-$ $r$ coefficients are calculated. Other elements operating continuously have $m+n-r$ coefficients of which at least one is different than zero.

Described algorithm is easy to be realized in some programming languages. For the given matrices A, B, $\mathbf{C}$ and $\mathbf{D}$, eigenvalues can be calculated and eliminated ones with value zero. Afterwards, the particular solution can be found as reduced row echelon form of the equation (17). In this paper we used Python 2.7 to compute matrix obtained via reduced row echelon form with the vector consisting of indices of leading 1 . Of course in discontinuous mode or in marginally stable continuous conduction mode there would be $r$ rows completely equal to zero, but for other state variables particular solution is provided as the value in the last column pointed by corresponding index. Zero rows are at the end of the matrix. In the programs for elements corresponding to the "skipped" indices are assigned values from the end of the previous time interval.

After determining coefficients of the homogeneous solution (21) and particular solution (17), the overall solution can be determined as

$$
\dot{\mathbf{x}}(t)=\mathbf{N}\left[\begin{array}{c}
\underline{\mathbf{x}}_{1} \\
\underline{\mathbf{x}}_{2} \\
\vdots \\
\underline{\mathbf{x}}_{m+n-q}
\end{array}\right]+\mathbf{x}_{p}
$$

with matrix $\mathbf{N}_{(m+n) \times(m+n)(m+n-q)}$, where $q=0$ if matrix $\mathbf{A}$ is nonsingular and $q=r$ if matrix $\mathbf{A}$ has $r$ zero eigenvalues, equal to

$$
\mathbf{N}=\left[\begin{array}{llll}
\mathbf{N}_{1} & \mathbf{N}_{2} & \ldots & \mathbf{N}_{m+n-q}
\end{array}\right]
$$

and

$$
\mathbf{N}_{i}=\operatorname{diag}\left\{\mathrm{e}{\underline{\lambda_{i}}}^{t}\right\}
$$

\subsection{Regulator Design}

The regulator generates a PWM signal which controls the controllable switch. Duty-ratio of the PWM signal is calculated inside regulator and provides exact moment of the transition between on-state and off-state of the controlled switch or switches. The exact time of the state transition is determined by the use of cutset algorithm with given absolute and relative errors.

Since the simulation program is a natural environment for the implementation of digital and/or discrete regulators, the regulator is completely designed in discrete manner. With a given Laplace transfer function which describes the dynamic behavior of an analog regulator, the discretization is done using pole-zero matching method. General form of the regulator transfer function in analog domain is given by

$G_{c}(s)=\frac{d(s)}{e(s)}=\frac{a_{c, n} s^{n}+a_{c, n-1} s^{n-1}+\cdots+a_{c, 0} s^{0}}{b_{c, m} s^{m}+b_{c, m-1} s^{m-1}+\cdots+b_{c, 0} s^{0}}$ 


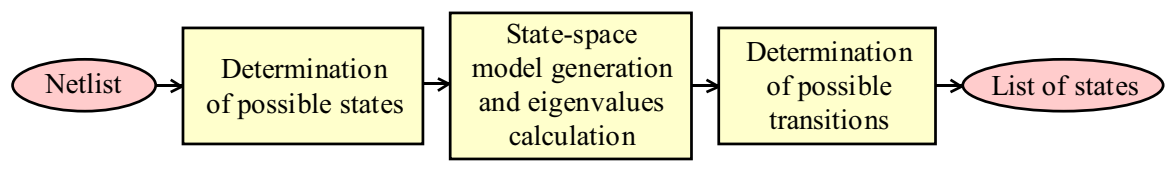

Fig. 4. Initialization for transient simulation flowchart.

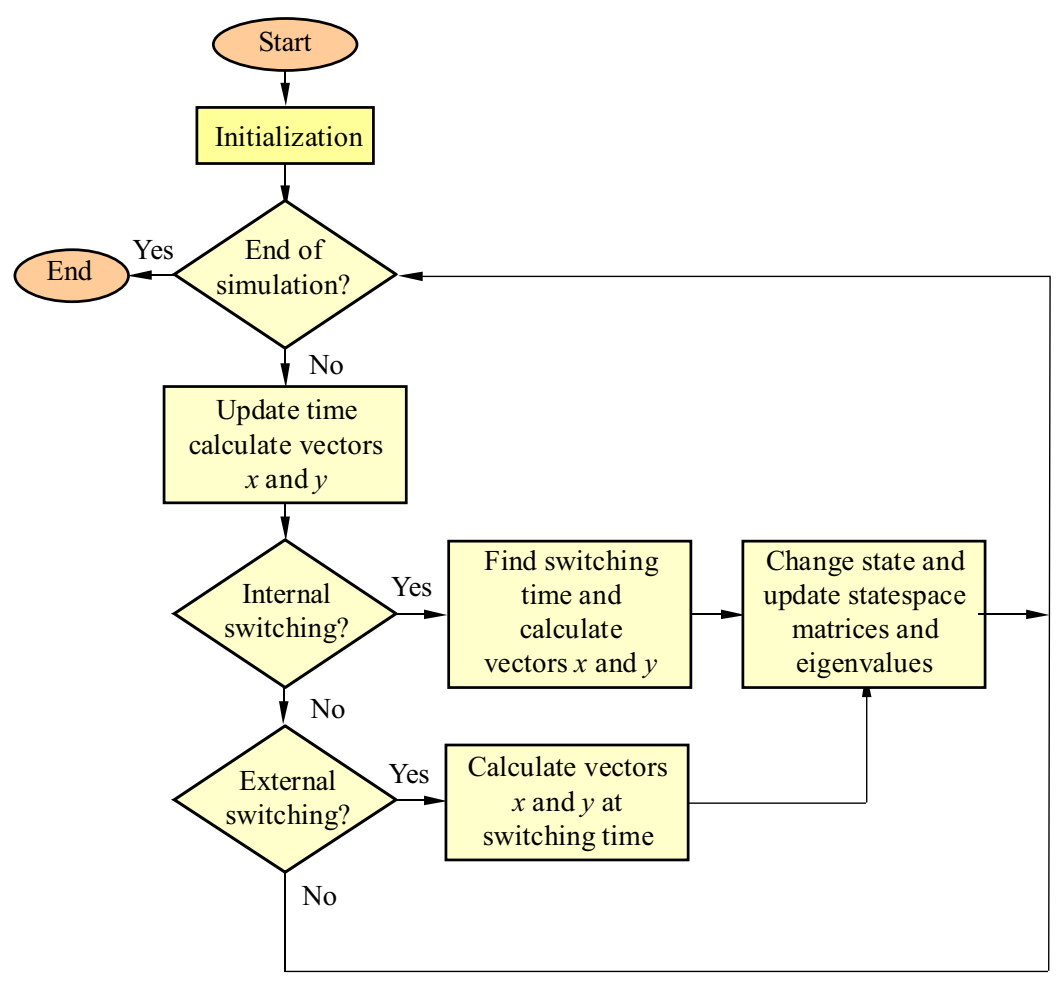

Fig. 5. Simulation flowchart

where $d(s)$ represents duty-ratio and $e(s)$ is the output error in the Laplace domain. Equation (25) can be rewritten as

$$
G_{c}(s)=\frac{d(s)}{e(s)}=G_{c 0} \frac{\prod_{i=1}^{n}\left(1+\frac{s}{s_{z i}}\right)}{\prod_{j=1}^{m}\left(1+\frac{s}{s_{p j}}\right)}
$$

where, $n$ is the order of nominator and $m$ is the order of denominator. Pole-zero matching transformation, $G_{c}(s) \rightarrow G_{d}(z)$, is done as follows [12]

- Finite poles and zeros of continuous transfer function $G_{c}(s), s_{p / z, i}=s_{\text {real }, i}+j s_{\text {imag }, i}$, are transformed into their discrete equivalent by using $z_{p / z, i}=$ $\mathrm{e}^{\left(s_{\text {real }, i}+j s_{\text {imag }, i}\right) T_{s}}$, where $T_{s}$ represents sampling period.

- Zeros at $s_{z, i} \rightarrow \infty$ are mapped into $z_{z, i}=-1$.

- DC gain of discrete transfer function is set to be equal to the DC gain of continuous transfer function $\mid G_{c}(s=$ $j \cdot 0)|=| G_{d}(z=1+j \cdot 0) \mid$.

Hence, using the obtained discrete transfer function, difference equation (or recurrent equation) for the duty-ratio determination is derived in a simple manner as

$$
G_{d}(z)=\frac{d(z)}{e(z)}=\frac{\sum_{l=0}^{i} a_{d, l} z^{l}}{\sum_{k=0}^{j} b_{d, k} z^{k}}=\frac{\sum_{l=0}^{i} \frac{a_{d, l}}{b_{d, j}} z^{l-j}}{1+\sum_{k=0}^{j-1} \frac{b_{d, k}}{b_{d, j}} z^{k-j}}
$$

with the nominator of degree $i$ and the denominator of degree $j$.

$$
d[n]=-\sum_{k=0}^{j-1} \frac{b_{d, k}}{b_{d, j}} d[n+k-j]+\sum_{l=0}^{i} \frac{a_{d, l}}{b_{d, j}} e[n+l-j] .
$$

For the desired regulator, values of errors and duty-ratio are updated once during switching period. Converter output values, which are same as regulator input values, at the end of the switching period $t=n T_{S}$ are sufficient for that purpose. Those values are already known, calculated, and thus new value for duty-ratio is easily obtained.

\subsection{Switching time determination}

DC-DC converters allow two switching types: internal, which occurs in case when condition for entering discontinuous mode is satisfied, and external triggered by the regulator, for which exact time of the transition and state variables' and output variables' values have to be known. An algorithm used for internal switching time determination relies on second-order Newton's interpolation method. External switching is closely related to the regulator design. 

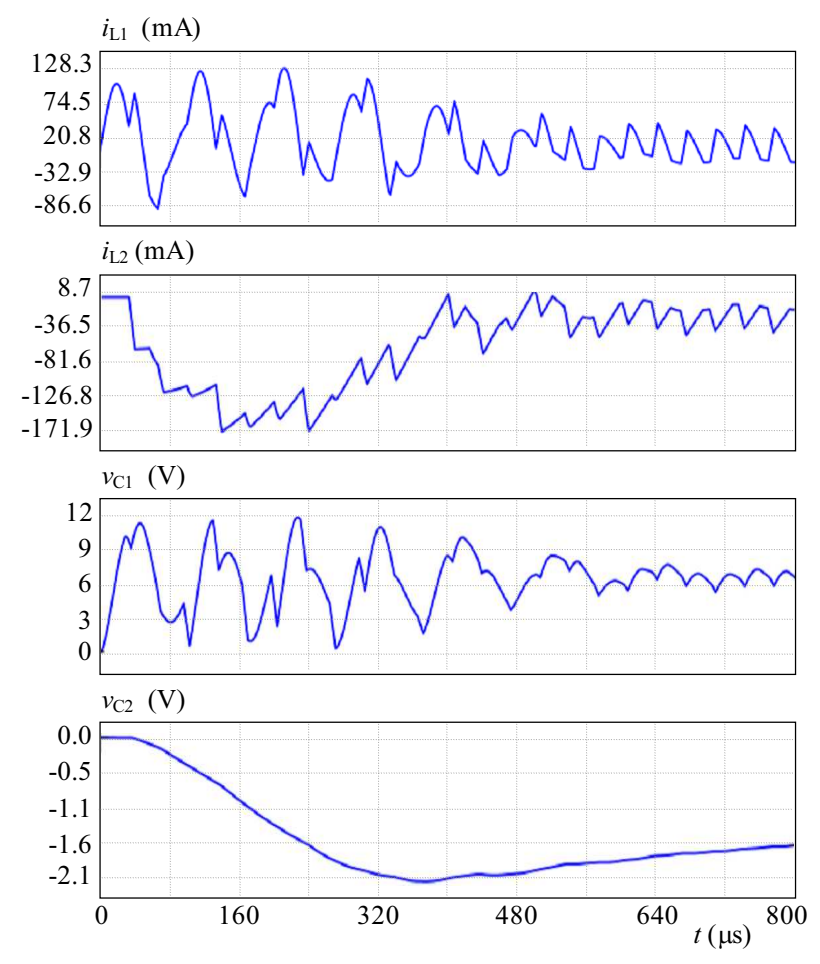

Fig. 6. Transient diagrams of state variables of the Ćuk converter shown in Fig. 3 for the first $800 \mu$ s and $d=0.2$

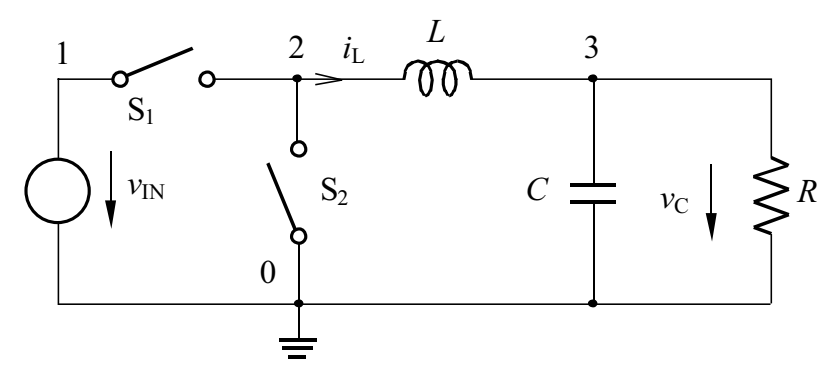

Fig. 8. Synchronous buck converter

\section{Internal switching}

Function that describes internal switching has the following form

$$
f(\mathbf{x})=\boldsymbol{\alpha} \mathbf{x}+\boldsymbol{\beta} \mathbf{u},
$$

where $\boldsymbol{\alpha}$ and $\boldsymbol{\beta}$ are vectors consisting of values 0,1 and -1 . Those values are coefficients that contain information for state variables and independent sources in equation (6) or (7) and depend on the determined discontinuous mode.

According to the second-order Newton's interpolation method, time is determined as

$$
t_{n+1}=t_{n}+\frac{1}{\frac{f^{(2)}\left(\mathbf{x}_{n}\right)}{2 f\left(\mathbf{x}_{n}\right)}-\frac{\dot{f}\left(\mathbf{x}_{n}\right)}{f\left(\mathbf{x}_{n}\right)}} .
$$

Matrix form of state-space model is very useful in this case, because

$$
\dot{f}\left(\mathbf{x}_{n}\right)=\boldsymbol{\alpha} \mathbf{A} \mathbf{x}_{n}+\boldsymbol{\alpha} \mathbf{B u}
$$

and

$$
f^{(2)}\left(\mathbf{x}_{n}\right)=\boldsymbol{\alpha} \mathbf{A}^{2} \mathbf{x}_{n}+\boldsymbol{\alpha} \mathbf{A B} \mathbf{u} .
$$

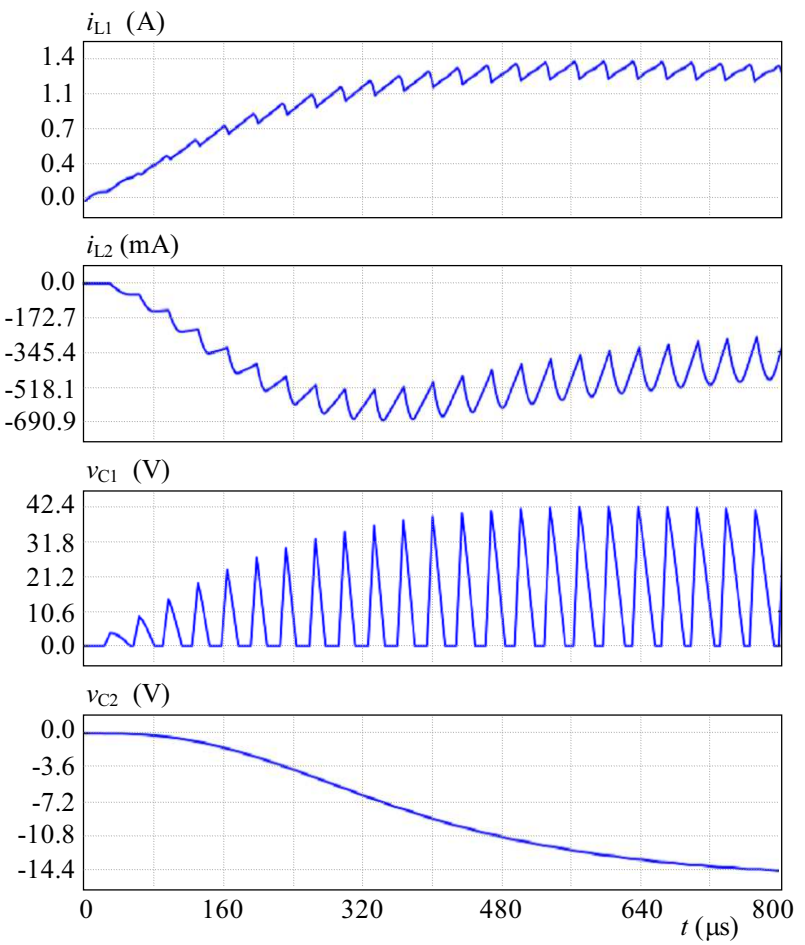

Fig. 7. Transient diagrams of state variables of the Ćuk converter shown in Fig. 3 for the first $800 \mu$ s and $d=0.8$

This method is used because it converges fast and is suitable for matrix state-space system's representation [4].

\section{External switching}

According to the regulator design, duty-ratio and error are calculated once during the switching period. On the other hand, the condition for state change caused by the regulator has to be checked after every time interval (switching period is divided into smaller time intervals). Checking is done based on whether the controlled switch or switches turns on or off. In both cases if the switching occurs a new point with exact switching time in which the state change occurs must be added and all state variable values must be calculated.

\section{Program implementation}

Using the constraints explained in Section 2, a program is implemented in Python 2.7 programming language. Program reads input netlist whose form is provided in Appendix 1. At the beginning of the simulation, the state-space model for each possible mode of operation is created and stored. After that the calculation of the transient begins during which the simulation results are stored. In Fig. 4, the simulation initialization is described. As can be seen from Fig. 4, the state-space matrices are calculated for every possible combination of the conducting switches. Then, the no valid states are eliminated. If in the circuit exists a DICM cutset or a DCVM loop then a discontinuous mode (DICM or DCVM) is possible and that state is saved. Afterwards for each possible state, eigenvalues are calculated and stored. 
With determined possible states and transitions and also calculated matrices and eigenvalues, simulation proceeds starting at time $t=0$ with given initial values for the state variables. The simulation starts with the initial state and continues with the same procedure until it reaches the stop time.

Step interval is 1000 points per period by default, but precision can be given as an input to the program. After each step interval, it is checked if there can be changes of state either by the regulator (limited duty-ratio) or by some of discontinuities inside the converter. In the case when a change occurs, by intersect algorithm, accurately a moment when the transition occurs is determined. This procedure is presented in Fig. 5 by the flowchart.

\section{Application Examples}

In this section, two simulation examples are provided. First example is the Cuk converter operating in discontinuous modes with a constant duty-ratio. This example proves that this simulator works for cases in which the system matrix is singular. The second example is the Buck converter with the regulator.

For the example of the Cuk converter in the open-loop configuration parameters $v_{I N}=5 \mathrm{~V}, L_{1}=645.4 \mu \mathrm{H}$, $L_{2}=996.3 \mu \mathrm{H}, C_{1}=217 \mathrm{nF}, C_{2}=14.085 \mu \mathrm{F}, R=$ $43 \Omega, f_{S}=30 \mathrm{kHz}$ are taken. Time domain diagrams are obtained as a result of detailed simulation during first $0.8 \mathrm{~ms}$ using calculated state-space matrices provided in Appendix 2. In Fig. 6, the Ćuk converter which for some intervals of time operates in DICM with constant dutyratio $d=0.2$, is shown. The converter operates in DICM after first $580 \mu \mathrm{s}$.

The simulation of the same Ćuk converter in an openloop configuration with constant duty-ratio $d=0.8$ provides converter operating in DCVM for some intervals of time during the switching period. In Fig. 7, it can be seen that the simulator successfully simulates the converter operating in DCVM. Both simulations of the Ćuk converter

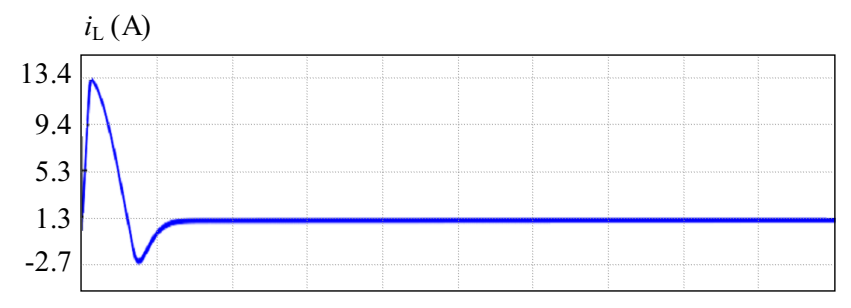

$$
v_{\mathrm{C}}(\mathrm{V})
$$

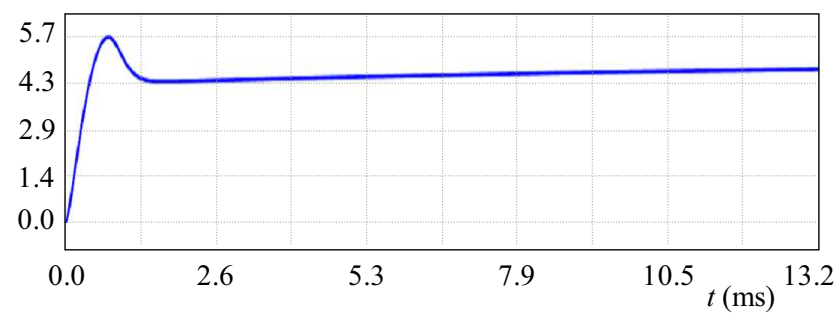

Fig. 9. Transient diagrams of state variables of the synchronous buck converter shown in Fig. 8 for the first $13.2 \mathrm{~ms}$ whose results are shown in Figs. 6 and 7 are verified using PLECS [20] and GeckoCIRCUITS [21]. Originally simulations were done with 100 points per period, but the number of points can be decreased to 30 points per period with the same simulation results.

The second example is the Buck converter. The first simulation is done for the synchronous Buck converter (realized using four-quadrant switches) depicted in Fig. 8 with parameters $v_{I N}=20 \mathrm{~V}, L=200 \mu \mathrm{H}, C=1 \mathrm{mF}$, $R=5 \Omega$ and $f_{S}=100 \mathrm{kHz}$, whose input netlist is in Appendix 1 and system's matrices in Appendix 3, with the regulator defined using the following transfer function

$$
H(s)=\frac{1.05 \cdot 10^{6} s^{2}+3.193 \cdot 10^{9} s+3.161 \cdot 10^{11}}{3.011 \cdot 10^{5} s^{2}+1.076 \cdot 10^{10} s}
$$

where the error signal is defined in comparison to reference voltage $e=v_{C}-5 \mathrm{~V}$. Simulation results are shown in Fig. 9.

The second case is when the Buck converter is realized using a switch and a diode as depicted in Fig. 2 with parameters $v_{I N}=10 \mathrm{~V}, L=25 \mu \mathrm{H}, C=330 \mu \mathrm{F}, R=$ $15 \Omega$ and $f_{S}=100 \mathrm{kHz}$. The converter is controlled with the regulator whose transfer function is

$$
H(s)=\frac{0.06 s+90}{s}
$$

and error signal defined as $e=v_{C}-5 \mathrm{~V}$. This converter operates in DICM for some intervals of time in the period, which can be seen in Fig. 10. Again, all simulation results are verified using simulators PLECS and GeckoCIRCUITS.

\section{Conclusion}

In this paper, an algorithm for simulation of DC-DC converters based on exact solutions of the circuits' equations, is provided. DC-DC converters' equations are written in the matrix form and solved as ordinary differential

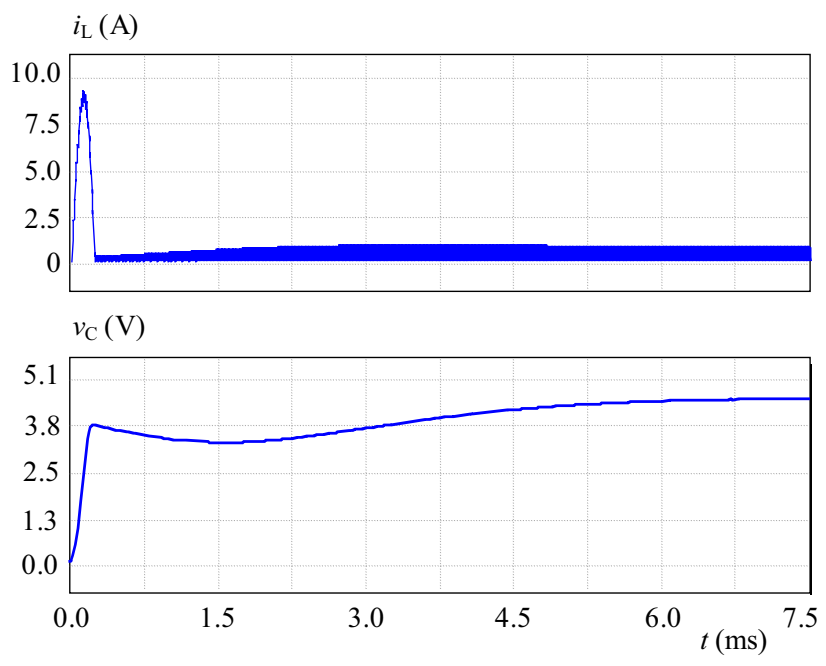

Fig. 10. Transient diagrams of state variables for buck converter shown in Fig. 2 for the first $7.5 \mathrm{~ms}$ 
equations by calculating their homogeneous and particular solutions. For the converter, a discrete regulator is designed which controls the switches.

The proposed algorithm is implemented in Python 2.7 programming language which is a free software. It is shown to have high accuracy by decreasing the number of calculation points. The simulation results for the two DC-DC converters of practical importance are provided. Furthermore, these simulation results verify the performance of the algorithm for each operating mode of the converter.

\section{Appendix A: Input netlist example}

Here, the input netlist for the synchronous Buck converter with parameters $v_{I N}=20 \mathrm{~V}, L=200 \mu \mathrm{H}, C=$ $1 \mathrm{mF}, R=5 \Omega$ and $f_{S}=100 \mathrm{kHz}$ is provided.

model = System.System()

\# SYSTEM DESCRIPTION

model.create_element (System.V(1, 1, 0, 20.0))

model.create_element (System.SW(1, 3, 1, 2))

model.create_element (System.SW(2, 3, 2, 0))

model.create_element (System.L (1, 2, 3, 200e-6))

model.create_element (System.C (1, 3, 0, 1e-3))

model.create_element (System.R(1, 3, 0, 5.0))

model.set_control (1, 'on_state')

model.set_control (2, 'off_state')

model.set_output ([ ['node', 3]])

controller $=$ Controller. Controller $([1],[2], \backslash$

'pole_zero_matching', [[[1.05e06, 3.193e09, ।

$3.161 \mathrm{e} 11],[3.011 \mathrm{e} 05,1.076 \mathrm{e} 10,0], 5]], 100 \mathrm{e} 3)$

simulation_time $=10 e-3$

Appendix B: Ćuk converter

For the Ćuk converter depicted in Fig. 3, the calculated state-space matrices (matrices' index is switches' state in order $(\mathrm{S} 2, \mathrm{~S} 1)$ ) are given below. Matrices C and D are the same for all of the states and therefore they are given just once.

$$
\begin{aligned}
\mathbf{A}_{(0,0)}= & {\left[\begin{array}{cccc}
0 & 0 & -\frac{1}{L_{1}+L_{2}} & -\frac{1}{L_{1}+L_{2}} \\
0 & 0 & -\frac{1}{L_{1}+L_{2}} & -\frac{1}{L_{1}+L_{2}} \\
0 & \frac{1}{C_{1}} & 0 & 0 \\
0 & \frac{1}{C_{2}} & 0 & -\frac{1}{C_{2} R}
\end{array}\right], \mathbf{B}_{(0,0)}=\left[\begin{array}{c}
\frac{1}{L_{1}+L_{2}} \\
\frac{1}{L_{1}+L_{2}} \\
0 \\
0
\end{array}\right] } \\
\mathbf{A}_{(0,1)} & =\left[\begin{array}{cccc}
0 & 0 & 0 & 0 \\
0 & 0 & -\frac{1}{L_{2}} & -\frac{1}{L_{2}} \\
0 & \frac{1}{C_{1}} & 0 & 0 \\
0 & \frac{1}{C_{2}} & 0 & -\frac{1}{C_{2} R}
\end{array}\right], \mathbf{B}_{(0,1)}=\left[\begin{array}{c}
\frac{1}{L_{1}} \\
0 \\
0 \\
0
\end{array}\right] \\
\mathbf{A}_{(1,0)}= & {\left[\begin{array}{cccc}
0 & 0 & -\frac{1}{L_{1}} & 0 \\
0 & 0 & 0 & -\frac{1}{L_{2}} \\
\frac{1}{C_{1}} & 0 & 0 & 0 \\
0 & \frac{1}{C_{2}} & 0 & -\frac{1}{C_{2} R}
\end{array}\right], \mathbf{B}_{(1,0)}=\left[\begin{array}{c}
\frac{1}{L_{1}} \\
0 \\
0 \\
0
\end{array}\right] }
\end{aligned}
$$

$$
\begin{aligned}
\mathbf{A}_{(1,1)} & =\left[\begin{array}{cccc}
0 & 0 & 0 & 0 \\
0 & 0 & 0 & -\frac{1}{L_{2}} \\
0 & 0 & 0 & 0 \\
0 & \frac{1}{C_{2}} & 0 & -\frac{1}{C_{2} R}
\end{array}\right], \mathbf{B}_{(1,1)}=\left[\begin{array}{c}
\frac{1}{L_{1}} \\
0 \\
0 \\
0
\end{array}\right] \\
\mathbf{C} & =\left[\begin{array}{llll}
0 & 0 & 0 & 1
\end{array}\right], \quad \mathbf{D}=[0]
\end{aligned}
$$

State variables' vector, independent source's vector and output variable's vector are given in the following forms

$$
\mathbf{x}=\left[\begin{array}{c}
i_{L 1} \\
i_{L 2} \\
v_{C 1} \\
v_{C 2}
\end{array}\right], \quad \boldsymbol{u}=\left[v_{I N}\right], \quad \boldsymbol{y}=\left[v_{C 2}\right]
$$

\section{Appendix C: Buck converter}

For the Buck converter depicted in Fig. 2 and in Fig. 8, the state-space matrices are

$$
\begin{gathered}
\mathbf{A}_{(0,0)}=\left[\begin{array}{cc}
0 & 0 \\
0 & -\frac{1}{C R}
\end{array}\right], \quad \mathbf{B}_{(0,0)}=\left[\begin{array}{l}
0 \\
0
\end{array}\right], \\
\mathbf{C}_{(0,0)}=\left[\begin{array}{ll}
0 & 1
\end{array}\right], \quad \mathbf{D}_{(0,0)}=[0]
\end{gathered}
$$

when switch $\mathrm{S} 1$ is off and diode $\mathrm{S} 2$ is off. This state doesn't occur in the synchronous Buck converter because it has only four-quadrant switches which can handle both polarities of the currents and voltages.

$$
\begin{gathered}
\mathbf{A}_{(0,1)}=\left[\begin{array}{cc}
0 & -\frac{1}{L} \\
\frac{1}{C} & -\frac{1}{C R}
\end{array}\right], \quad \mathbf{B}_{(0,1)}=\left[\begin{array}{c}
\frac{1}{L} \\
0
\end{array}\right], \\
\mathbf{C}_{(0,1)}=\left[\begin{array}{ll}
0 & 1
\end{array}\right], \quad \mathbf{D}_{(0,1)}=[0]
\end{gathered}
$$

when switch $\mathrm{S} 1$ is on and diode/switch S2 is off and

$$
\begin{gathered}
\mathbf{A}_{(1,0)}=\left[\begin{array}{cc}
0 & -\frac{1}{L} \\
\frac{1}{C} & -\frac{1}{C R}
\end{array}\right], \quad \mathbf{B}_{(1,0)}=\left[\begin{array}{l}
0 \\
0
\end{array}\right], \\
\mathbf{C}_{(1,0)}=\left[\begin{array}{ll}
0 & 1
\end{array}\right], \quad \mathbf{D}_{(1,0)}=[0]
\end{gathered}
$$

when switch $\mathrm{S} 1$ is off and diode/switch $\mathrm{S} 2$ is on. It can be seen that when both switches are not conducting inductor current falls to zero and thus discontinuous inductor current mode occurs. State when both semiconductors are on is not valid.

State variables' vector, independent source's vector and output variable's vector are given in the following forms

$$
\mathbf{x}=\left[\begin{array}{c}
i_{L} \\
v_{C}
\end{array}\right], \quad \boldsymbol{u}=\left[v_{I N}\right], \quad \boldsymbol{y}=\left[v_{C}\right]
$$




\section{REFERENCES}

[1] D. Maksimović, A. M. Stanković, V. J. Thottuvelil and G. C. Verghese, "Modeling and Simulation of Power Electronic Converters", Proceedings of the IEEE, vol. 89, no. 6, 898-912, 2001.

[2] R. W. Erickson and D. Maksimovic," Fundamentals of Power Electronics, Springer Science \& Business Media, 2007.

[3] L. W. Nagel and D. O. Pederson," SPICE: Simulation Program with Integrated Circuit Emphasis, Electronics Research Laboratory, College of Engineering, University of California, 1973.

[4] D. Li, R. Tymerski an T. Ninomiya, "PECS - an Efficient Solution for Simulating Switched Networks with Nonlinear Elements", IEEE Transactions on Industrial Electronics, vol. 48, no. 2, 367-376, 2001.

[5] R. Tymerski and V. Vorperian, "Generation, Classification and Analysis of Switched-Mode DC-to-DC Converters by the Use of Converter Cells", INTELEC'86. International Telecommunications Energy Conference, IEEE, 1986, 181-195.

[6] D. Maksimovic, Synthesis of PWM and Quasi-Resonant DC-to -DC Power Converters, PhD Dissertation, California Institute of Technology, 1200 E California Blvd, Pasadena, CA 91125, United States, 1989.

S. Ben-Yaakov, "Average Simulation of PWM Converters by Direct Implementation of Behavioural Relationships", International journal of electronics, vol. 77, no. 5, 731-746, 1994.

[8] J. Sun, D. M. Mitchell, M. F. Greuel, P. T. Krein and R. M. Bass, "Averaged Modeling of PWM Converters Operating in Discontinuous Conduction Mode", IEEE Transactions on Power Electronics, vol. 16, no. 4, 482-492, 2001.

[9] A. Davoudi, J. Jatskevich and T. Rybel, "Numerical State-Space Average-Value Modeling of PWM DC-DC Converters Operating in DCM and CCM", IEEE Transactions on Power Electronics, vol. 21, no. 4, 1003-1012, 2006.

[10] A. Davoudi and J. Jatskevich, "Realization of Parasitics in State -Space Average-Value Modeling of PWM DC-DC Converters", IEEE Transactions on Power Electronics, vol. 21, no. 4, 1142$1147,2006$.

[11] R. A. Horn and C. R. Johnson, Matrix Analysis, Cambridge university press, 2012.

[12] A. Prodic, D. Maksimovic and R. W. Erickson, "Design and Implementation of a Digital PWM Controller for a High-Frequency Switching DC-DC Power Converter", The 27 th Annual Conference of the IEEE Industrial Electronics Society, 2001 IECON'01, vol. 2, IEEE,2001,893-898.
[13] D. Maksimović and S. Ćuk, "A General Approach to Synthesis and Analysis of Quasi-Resonant Converters", 20 th Annual IEEE Power Electronics Specialists Conference, 1989. PESC'89 Record, IEEE, 1989, 713-727.

[14] K. Schenk and S. Ćuk, "Small Singnal Analysis of Converters with Multiple Discontinuous Conduction Modes", IEEE Power Electronics Specialists Conference, Institute of Electrical Engineers Inc (IEE), 1998, 623-629.

[15] D. Maksimović and S. Ćuk, "A Unified Analysis of PWM Converters in Discontinuous Modes", IEEE Transactions on Power Electronics, vol. 6, no. 3, 476-490, 1991.

[16] M. Zhu, F. L. Luo and Y. He, "Remaining Inductor Current Phenomena of Complex DC-DC Converters in Discontinuous Conduction Mode: General Concepts and Case Study", IEEE Transactions on Power Electronics, vol. 23, no. 2, 1014-1019, 2008.

[17] C.-W. Ho, A. Ruehli and P. Brennan, "The Modified Nodal Approach to Network Analysis", IEEE Transactions on Circuits and Systems, vol. 22, no. 6, 504-509, 1975.

[18] R. D. Middlebrook and S. Ćuk, "A General Unified Approach to Modelling Switching-Converter Power Stages", Power Electronics Specialists, 18-34 Conference, 1976, IEEE, 1976.

[19] R. Erickson, "Synthesis of Switched-Mode Converters", Power Electronics Specialists Conference, IEEE, 1983, 9-22.

[20] J. Alimeling and W. P. Hammer, "PLESC-Piece-Wise Linear Electrical Circuit Simulation for Simulink", Power Electronics and Drive Systems, PEDS'99. Proceedings of the IEEE 1999 International Conference on, vol. 1, IEEE, 1999, 355-360.

[21] A. Müsing and J. W. Kolar, "Successful Online Education-Geckocircuits as Open-Source Simulation Platform", 2014 International Power Electronics Conference (IPEC-Hiroshima 2014-EC CE ASIA), IEEE, 2014, 821-828.

Received 28 October 2016

Aleksandra Lekić is born in Belgrade, Serbia, in 1989. She received BS and MS degrees in Electrical Engineering from the University of Belgrade, in 2012 and 2013, respectively. Currently, she is a final year student of $\mathrm{PhD}$ studies at the same school. Since December 2012, she works as a teaching assistant at the School of Electrical Engineering, University of Belgrade. The field of her interest is control in power electronics circuits. 